\title{
QUEEN'S
UNIVERSITY
BELFAST
}

\section{Experimental Realization of Dicke States of up to Six Qubits for Multiparty Quantum Networking}

Prevedel, R., Cronenberg, G., Tame, M., Paternostro, M., Walther, P., Kim, M., \& Zeilinger, A. (2009).

Experimental Realization of Dicke States of up to Six Qubits for Multiparty Quantum Networking. Physical Review Letters, 103(2), [020503]. https://doi.org/10.1103/PhysRevLett.103.020503

Published in:

Physical Review Letters

Document Version:

Publisher's PDF, also known as Version of record

Queen's University Belfast - Research Portal:

Link to publication record in Queen's University Belfast Research Portal

Publisher rights

(c) 2009 The American Physical Society

\section{General rights}

Copyright for the publications made accessible via the Queen's University Belfast Research Portal is retained by the author(s) and / or other copyright owners and it is a condition of accessing these publications that users recognise and abide by the legal requirements associated with these rights.

Take down policy

The Research Portal is Queen's institutional repository that provides access to Queen's research output. Every effort has been made to ensure that content in the Research Portal does not infringe any person's rights, or applicable UK laws. If you discover content in the Research Portal that you believe breaches copyright or violates any law, please contact openaccess@qub.ac.uk. 


\title{
Experimental Realization of Dicke States of up to Six Qubits for Multiparty Quantum Networking
}

\author{
R. Prevedel, ${ }^{1}$ G. Cronenberg, ${ }^{1}$ M. S. Tame, ${ }^{2}$ M. Paternostro, ${ }^{2}$ P. Walther, ${ }^{1,3}$ M. S. Kim, ${ }^{2}$ and A. Zeilinger ${ }^{1,3}$ \\ ${ }^{1}$ Faculty of Physics, University of Vienna, Boltzmanngasse 5, A-1090 Vienna, Austria \\ ${ }^{2}$ School of Mathematics and Physics, The Queen's University, Belfast, BT7 1NN, United Kingdom \\ ${ }^{3}$ Institute for Quantum Optics and Quantum Information (IQOQI), Austrian Academy of Sciences, \\ Boltzmanngasse 3, A-1090 Vienna, Austria \\ (Received 12 March 2009; published 10 July 2009)
}

\begin{abstract}
We report the first experimental generation and characterization of a six-photon Dicke state. The produced state shows a fidelity of $F=0.56 \pm 0.02$ with respect to an ideal Dicke state and violates a witness detecting genuine six-qubit entanglement by 4 standard deviations. We confirm characteristic Dicke properties of our resource and demonstrate its versatility by projecting out four- and five-photon Dicke states, as well as four-photon Greenberger-Horne-Zeilinger and $W$ states. We also show that Dicke states have interesting applications in multiparty quantum networking protocols such as open-destination teleportation, telecloning, and quantum secret sharing.
\end{abstract}

DOI: 10.1103/PhysRevLett.103.020503

Multipartite entanglement is at the core of studies probing the foundations of quantum physics and represents a key component in a wide range of quantum-information processing tasks [1]. So far, Greenberger-Horne-Zeilinger (GHZ) [2], $W$ [3], cluster, and graph states [4] have been studied and experimentally investigated [5]. However, other nonequivalent classes of quantum states with interesting symmetries exist [6]. In particular, Dicke states [7] provide a rich opportunity for exploring multipartite entanglement. Recent studies have focused on techniques for generating, detecting, and characterizing these states [8,9] in atomic, ion-trap, [10] and optical [11] settings.

In this Letter we report the experimental generation and investigation of a variety of multiphoton entangled states. We present a flexible linear-optics setup that can produce four-, five-, and six-photon representatives of the important class of Dicke states, as well as four-photon GHZ states. Information is encoded in the polarization degrees of freedom of entangled photons produced by high-order spontaneous parametric down-conversion (SPDC). We show that our generated states are genuinely multipartite entangled by using tailor-made and experimentally favorable witness tools. These new characterization methods are important in virtue of the nonideal nature of the six-photon state: although spurious nonlinear processes affect its quality, quantum features can still be observed and characterized. We also highlight the potential for quantum control in large Hilbert spaces by evaluating protocols such as opendestination teleportation, telecloning, and quantum secret sharing [11-15].

Experiment.-Figure 1(a) shows the setup for the generation of the three-excitation six-photon Dicke state $\left|D_{6}^{(3)}\right\rangle=\frac{1}{\sqrt{20}} \sum_{P}|H H H V V V\rangle_{123456}$. Here, $|H(V)\rangle_{i}$ are horizontal (vertical) polarization states of a photon in spatial mode $i=1, \ldots, 6$, which encode the logical states of a qubit, while $\sum_{P}$ denotes the sum over all permutations of logical states [16]. In the setup, six photons are probabil-
PACS numbers: 03.67.Bg, 03.67.Mn, 42.50.Dv, 42.50.Ex

istically distributed among the spatial modes by nonpolarizing beamsplitters (BSs): upon detecting one photon in each mode we postselectively observe $\left|D_{6}^{(3)}\right\rangle$. We use higher-order emissions of a collinear type-II SPDC process for the simultaneous production of three pairs of photons [17]. A Coherent Inc. Verdi V-18 laser is combined with a mode-locked Mira HP Ti:sapphire oscillator to reach the energy necessary to observe third-order SPDC emissions. The pulsed-laser output $(\tau=200 \mathrm{fs}, \quad \lambda=810 \mathrm{~nm}$, $76 \mathrm{MHz}$ ) is frequency doubled using a 2-mm-thick lithium triborate crystal, resulting in UV pulses of $1.4 \mathrm{~W} \mathrm{cw}-$ average. To avoid optical damage to the antireflection coating of the lithium triborate, we continuously translate it with a stepmotor, achieving a very stable source of UV pulses (power and count-rate fluctuations less than 1\%-2\% over $30 \mathrm{~h}$ ). The UV pulses are focused onto a 2-mm-thick $\beta$-barium borate (BBO) type-II crystal, cut for collinear SPDC. Dichroic mirrors separate the down-converted photons from the UV pump, and a compensator erases walkoff effects. We use high-transmittivity interference filters $(\Delta \lambda=3 \mathrm{~nm})$ to spatially and spectrally select the photons, which are coupled to a single-mode fiber guiding them to the Dicke setup of Fig. 1(a). At $1.4 \mathrm{~W}$ of UV pump power, we observe $\sim 0.003$ six-photon Dicke states per second. Higher power increases the sixfold rate while decreasing the fidelity due to undesired detection events from higherorder SPDC emissions [17].

State characterization.-In order to detect the presence of genuine multipartite entanglement (GME) in our experimental states, i.e., quantum entanglement shared by all the particles involved, we use collective-spin inequalities [9]. Various entanglement witnesses have been found to be well suited to the class of Dicke states [8]. They are experimentally appealing, due to the small number of local measurement settings (LMSs) required, in stark contrast to their more demanding projector-based counterparts. We start with the collective-spin witness $\left\langle\mathcal{W}^{s}\right\rangle_{f}=\left\langle J_{x}^{2}+J_{y}^{2}\right\rangle_{f}$, 


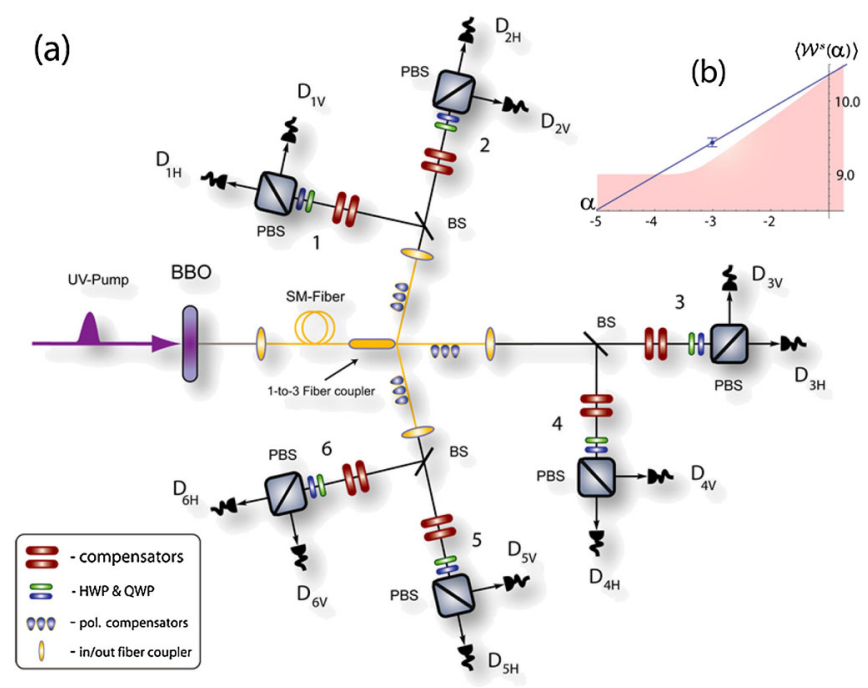

FIG. 1 (color online). (a) Setup for generating the six-photon Dicke state $\left|D_{6}^{(3)}\right\rangle$. Photons are distributed into modes $1, \ldots, 6$ via a 1-to-3 fiber coupler, followed by 50:50 BSs. The expected probability to find one photon in each spatial mode, corresponding to the state $\left|D_{6}^{(3)}\right\rangle$, is $p \sim 0.015$. The fiber and BSs introduce birefringence, compensated by fiber-squeezers and birefringent crystals. State characterization is performed via polarization analysis of sixfold coincidences by a quarter-wave plate (QWP), a half-wave plate (HWP), and a polarizing beam splitter (PBS), whose output ports are monitored by multimode fibercoupled single-photon detectors. Each detector signal enters a coincidence logic that records multiphoton coincidences. (b) Biseparability region (shaded) for $\left\langle W^{s}(\alpha)\right\rangle_{b s}$ and experimental point (predicted line) for $\left\langle\mathcal{W}^{s}(\alpha)\right\rangle_{\varrho_{6}^{(3)}}$.

where $f$ refers to the state over which the expectation value is calculated. Here, $J_{i}=\frac{1}{2} \sum_{k=1}^{N} \sigma_{i}^{(k)}(i=x, y, z)$ are collective-spin operators of $N$ qubits with label $k$, and $\sigma_{i}^{(k)}$ denotes the $i$ Pauli operator. By using the techniques described in Ref. [18], it can be seen that for any six-qubit biseparable $(b s)$ state $\left\langle\mathcal{W}^{s}\right\rangle_{b s} \leq 11.02$, so that $\left.\left\langle\mathcal{W}^{s}\right\rangle_{f}\right\rangle$ 11.02 will detect the presence of GME in $f$. However, due to the nonideal two-qubit correlations upon which $\mathcal{W}^{s}$ depends [shown in Figs. 2(a) and 2(b) for $\left\langle J_{x}^{2}\right\rangle$ and $\left\langle J_{y}^{2}\right\rangle$ ], our experimental state $\varrho_{6}^{(3)}$ gives $\left\langle\mathcal{W}^{s}\right\rangle_{\varrho_{6}^{(3)}}<11.02$. To obtain a witness that detects GME for a nonideal state, we insert a term proportional to $J_{z}^{2}$, for which $\left\langle J_{z}^{2}\right\rangle_{D_{6}^{(3)}}=0$. This gives the more general witness $\mathcal{W}^{s}(\alpha)=J_{x}^{2}+J_{y}^{2}+$ $\alpha J_{z}^{2}(\alpha \in \mathbb{R})$. We then search for values of $\alpha$ such that $\left.\left\langle\mathcal{W}^{s}(\alpha)\right\rangle_{\varrho_{0}^{(3)}}\right\rangle\left\langle\mathcal{W}^{s}(\alpha)\right\rangle_{b s}$. In Fig. 2(c) we show the twoqubit correlations for $\left\langle J_{z}^{2}\right\rangle_{\varrho_{0}^{(3)}}$, which contribute to $\left\langle\mathcal{W}^{s}(\alpha)\right\rangle$ shown in Fig. 1(b). A range of $\alpha$ exists where $\left.\left\langle\mathcal{W}^{s}(\alpha)\right\rangle_{\varrho_{6}^{(3)}}\right\rangle\left\langle\mathcal{W}^{s}(\alpha)\right\rangle_{b s}$ : the gap is optimized at $\alpha=$ -3 , where $\left\langle\mathcal{W}^{s}(\alpha)\right\rangle_{b s}^{\max }-\left\langle\mathcal{W}^{s}(\alpha)\right\rangle_{\varrho_{6}^{(3)}}=-0.24 \pm 0.06$, thus confirming GME for our experimental state.

We now further probe the features of $\varrho_{6}^{(3)}$ and consider the multiphoton correlator $\mathcal{C}_{i, j}^{\otimes N}(\theta)=\left(\cos \theta \sigma_{i}+\sin \theta \sigma_{j}\right)^{\otimes N}$. This allows the sampling of $N$-photon correlations in or-

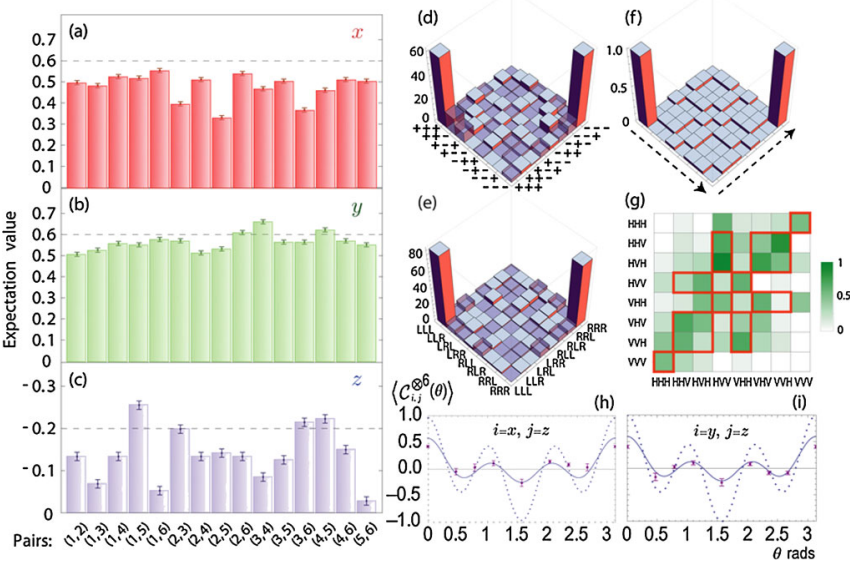

FIG. 2 (color online). Experimental study of the six-photon Dicke state $\rho_{6}^{(3)}$. (a)-(c) Correlations $\left\langle\sigma_{i}^{(j)} \sigma_{i}^{(k)}\right\rangle$ for qubit pairs $(j$, k) $(i=x, y, z)$ for $\left\langle W^{s}(\alpha)\right\rangle$. Dashed lines are ideal values. (d), (e), and (g) Coincidences for photons measured in $| \pm\rangle=(|H\rangle \pm$ $|V\rangle) / \sqrt{2}, \mid L$ or $R\rangle=(|H\rangle \pm i|V\rangle) / \sqrt{2}$, and $\mid H$ or $V\rangle$ (rescaled). (f) Ideal populations for (d) and (e). (h) and (i) Multiphoton correlations. Dashed (solid) lines are the patterns of $\left\langle\mathcal{C}_{x / y, z}^{\otimes 6}(\theta)\right\rangle$ for $\left|D_{6}^{(3)}\right\rangle\left(\rho_{\text {sim }}\right)$. The dots are experimental points.

thogonal planes of the single-qubit Bloch sphere, providing important information about the off-diagonal elements of the density matrix and thus its coherence properties. One finds $\left\langle\mathcal{C}_{i, z}^{\otimes 6}(\theta)\right\rangle_{D_{6}^{(3)}}=[3 \cos (2 \theta)+5 \cos (6 \theta)] / 8$, for $i=x$, $y$. Only the coherences within the Dicke state are responsible for the interference between the trigonometric functions in $\left\langle\mathcal{C}_{i, z}^{\otimes 6}(\theta)\right\rangle_{D_{6}^{(3)}}[17,19]$. In Figs. 2(h) and 2(i) we compare the ideal coherence signature with that of $\varrho_{6}^{(3)}$, finding a reduced visibility. We also compare $\varrho_{6}^{(3)}$ with the behavior of the state $\rho_{\text {sim }}$ resulting from a detailed simulation of our setup including multiple-pair emissions and losses [17]. The simulated state spans a Hilbert-space sector which is larger than the $2^{6}$-dimensional space of $\left|D_{6}^{(3)}\right\rangle$. Moreover, the presence of spurious state components in $\rho_{\text {sim }}$ affects the ideal populations and coherences, as shown in Ref. [17]. The accuracy of the simulation is confirmed by the behavior of $\left\langle\mathcal{C}_{i, j}^{\otimes 6}(\theta)\right\rangle_{\rho_{\text {sim }}}$ shown in Figs. 2(h) and 2(i), revealing good agreement with our data. Our analysis of $\varrho_{6}^{(3)}$ is strengthened by evaluating the state fidelity $\left\langle F_{D_{6}^{(3)}}\right\rangle_{Q_{6}^{(3)}}$, where the projector $F_{D_{6}^{(3)}}=$ $\left|D_{6}^{(3)}\right\rangle\left\langle D_{6}^{(3)}\right|$ is decomposed into 544 terms involving Pauli operators, requiring $21 \mathrm{LMSs}$ for their evaluation [20]. We find $\left\langle F_{D_{6}^{(3)}}\right\rangle_{\varrho_{6}^{(3)}}=0.56 \pm 0.02$, which agrees well with the value 0.61 from $\rho_{\text {sim }}$. The small discrepancy is due to slightly asymmetric fiber coupling of $\mid H$ or $V\rangle$ due to SPDC birefringence. The setup performances are thus limited by noise from higher-order emissions [17]. Despite such clearly consistent results, the measured fidelity prevents us from unambiguously claiming that our generated state is Dicke-class [21]. As full state tomography is experimentally prohibitive, we complement the fidelity analysis with additional characterization tools. 
We now explore the nested structure of Dicke states and their persistence of entanglement by conditionally generating four- and five-photon entangled states via projections of $\left|D_{6}^{(3)}\right\rangle[11,22]$. For example, by measuring one photon in $|H\rangle$, the five-photon state $\left|D_{5}^{(2)}\right\rangle[16]$ is projected out. This state is equivalent to $\sigma_{x}^{\otimes 5}\left|D_{5}^{(3)}\right\rangle$, showing that navigation through the Dicke class of states is possible via projections and local operations. Indeed, one can write $\left|D_{N}^{(m)}\right\rangle=$ $\left(C_{N}^{m}\right)^{-1 / 2}\left[\left(C_{N-1}^{m-1}\right)^{1 / 2}|H\rangle\left|D_{N-1}^{(m-1)}\right\rangle+\left(C_{N-1}^{m}\right)^{1 / 2}|V\rangle\left|D_{N-1}^{(m)}\right\rangle\right]$ and navigate as shown in Fig. 3(m). We start by experimentally projecting out the five-photon state $\varrho_{5}^{(2)}$ in modes 2,..,6 [Figs. 3(a)-3(f) show the experimental data]. For five-qubit states we have $\left\langle\mathcal{W}^{s}\right\rangle_{b s} \leq 7.87$ [18], giving $\left\langle\mathcal{W}^{s}\right\rangle_{b s}^{\max }-\left\langle\mathcal{W}^{s}\right\rangle_{\varrho_{5}^{(2)}}=-0.21 \pm 0.04$, thus detecting GME. To check consistency, we also projected photon 6 in $|H\rangle$, finding $\left\langle\mathcal{W}^{s}\right\rangle_{b s}^{\max }-\left\langle\mathcal{W}^{s}\right\rangle_{\varrho_{5}^{(2)}}=-0.32 \pm 0.02$.

Next, we project out the four-photon Dicke state $\left|D_{4}^{(2)}\right\rangle$ [16] by measuring one photon in $|H\rangle$ and another in $|V\rangle$. Using $\left\langle\mathcal{W}^{s}\right\rangle_{b s} \leq 5.23$ [8], the correlations for the experimental state $\varrho_{4}^{(2)}$ in modes $3, \ldots, 6$ [shown in Figs. 3(g)-3(1)] give $\left\langle\mathcal{W}^{s}\right\rangle_{b s}^{\max }-\left\langle\mathcal{W}^{s}\right\rangle_{\varrho_{4}^{(2)}}=-0.16 \pm 0.07$, thus detecting GME. Moreover, we have evaluated the state fidelity $\left\langle F_{D_{4}^{(2)}}\right\rangle_{\varrho_{4}^{(2)}}=0.66 \pm 0.05$ using 9 LMSs [23]. We complete our study of four-photon Dicke states by assessing a fourphoton $W$ state $\left|D_{4}^{(1)}\right\rangle$ (equivalent to $\sigma_{x}^{\otimes 4}\left|D_{4}^{(3)}\right\rangle$ ), generated
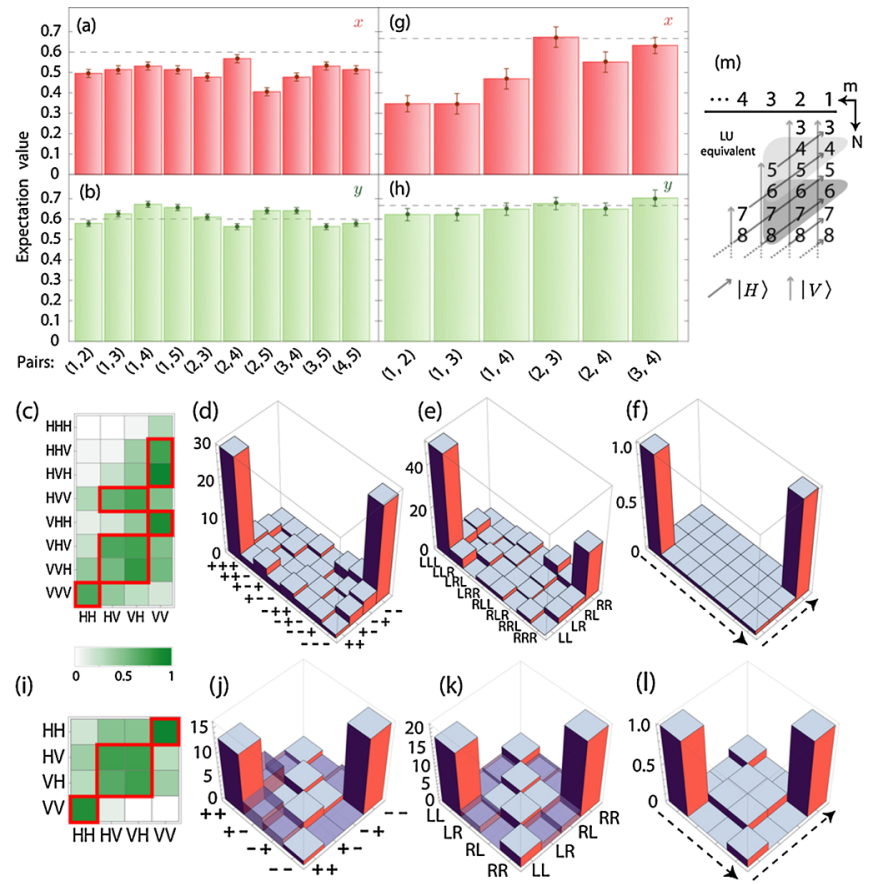

FIG. 3 (color online). Experimental data for the Dicke states $\varrho_{5}^{(2)}$ and $\varrho_{4}^{(2)}$. (a) and (b) [(g) and (h)] Correlations $\left\langle\sigma_{i}^{(j)} \sigma_{i}^{(k)}\right\rangle$ for qubit pairs $(j, k)$ of $\varrho_{5}^{(2)}\left(\varrho_{4}^{(2)}\right)$, with $i=x, y$. (c)-(e) [(i)(k)] Coincidences for the five (four) photons measured in $\mid H$ or $V\rangle,| \pm\rangle$, and $\mid L$ or $R\rangle$. (f) [(1)] Ideal populations for $| \pm\rangle$ and $\mid L$ or $R\rangle$. (m) Navigating the Dicke class by measurement. from $\left|D_{6}^{(3)}\right\rangle$ upon measurement of two photons in $|H\rangle$. Experimentally, we project the state $\varrho_{4}^{(1)}$ into modes $3, \ldots, 6$ [coincidence counts shown in Figs. 4(a)-4(d)]. Although $\left|D_{4}^{(1)}\right\rangle$ does not exceed $\left\langle\mathcal{W}^{s}\right\rangle_{b s}^{\max }$, for our experimental state we find $\left\langle\mathcal{W}^{s}\right\rangle_{b s}^{\max }-\left\langle\mathcal{W}^{s}\right\rangle_{\varrho_{4}^{(1)}}=-0.2 \pm 0.1$ due to $\left\langle J_{x, y}^{2}\right\rangle$ being slightly larger than their ideal values, thus detecting GME. We further characterize $\varrho_{4}^{(1)}$ by evaluating $\left\langle F_{D_{4}^{(1)}}\right\rangle_{\varrho_{4}^{(1)}}=0.62 \pm 0.02$, using 7 LMSs [24]. Finally, a state locally equivalent to $\left|\mathrm{GHZ}_{4}\right\rangle=(1 / \sqrt{2}) \times$ $\left[|H\rangle^{\otimes 4}+|V\rangle^{\otimes 4}\right]$ can also be generated from $\left|D_{6}^{(3)}\right\rangle$ by measuring one photon in $|+\rangle$ and another in $|-\rangle$. Ideally, this produces $\left(\left|D_{4}^{(1)}\right\rangle-\left|D_{4}^{(3)}\right\rangle\right) / \sqrt{2} \equiv$ $\sigma_{z}^{(1)}\left(\mathcal{H} \sqrt{\sigma_{z}}\right)^{\otimes 4}\left|\mathrm{GHZ}_{4}\right\rangle$ ( $\mathcal{H}$ is the Hadamard gate). The state fidelity (using $5 \mathrm{LMSs}$ ) is $\left\langle F_{\mathrm{GHZ}_{4}}\right\rangle_{\varrho_{\mathrm{GHZ}}}=0.56 \pm$ 0.02 , giving a projector-based witness value of $\langle\mathcal{W}\rangle_{\mathrm{GHZ}}=$ $-0.06 \pm 0.02$ [24], thus confirming GME.

Quantum protocols. - Despite the nonideal value of the state fidelity, the symmetries within our six-photon resource make it suitable for several key quantumnetworking protocols $[11,14]$, some of which have been demonstrated in four-photon settings. Tracing out $N-2$ qubits, one finds the two-photon state $\rho=\alpha_{N}\left|\psi^{+}\right\rangle \times$ $\left\langle\psi^{+}\right|+\left(1-\alpha_{N}\right)[|H H\rangle\langle H H|+| V V\rangle\langle V V|] / 2$ with $\alpha_{N}=$ $N /[2(N-1)]$ for $N \geq 4$ [9] and $\left|\psi^{ \pm}\right\rangle=(|H V\rangle \pm$ $|V H\rangle) / \sqrt{2}$. Here, the maximal singlet fraction $F_{\mathrm{msf}}$ [25], given by the maximum of $\left\langle F_{\psi^{-}}\right\rangle$under local operations and classical communication, helps in assessing the usefulness of $\rho$ for networking tasks. We consider using $\rho$ as a teleportation channel $[11,14]$, where the maximum fidelity achievable for teleporting an arbitrary state is $F_{\max }=$ $\left(2 F_{\mathrm{msf}}+1\right) / 3$. For $\left|D_{N}^{(N / 2)}\right\rangle, F_{\mathrm{msf}}=\alpha_{N}$; thus $F_{\max }=$ $\frac{2 N-1}{3(N-1)}$. Figure 4(e) shows $F_{\max }$ for all pairs of photons from $\varrho_{6}^{(3)}$ and the ideal value 0.73 (upper dashed line). As any photon pair in $\left|D_{6}^{(3)}\right\rangle$ provides a channel for teleportation, regardless of operations applied to the others, one can
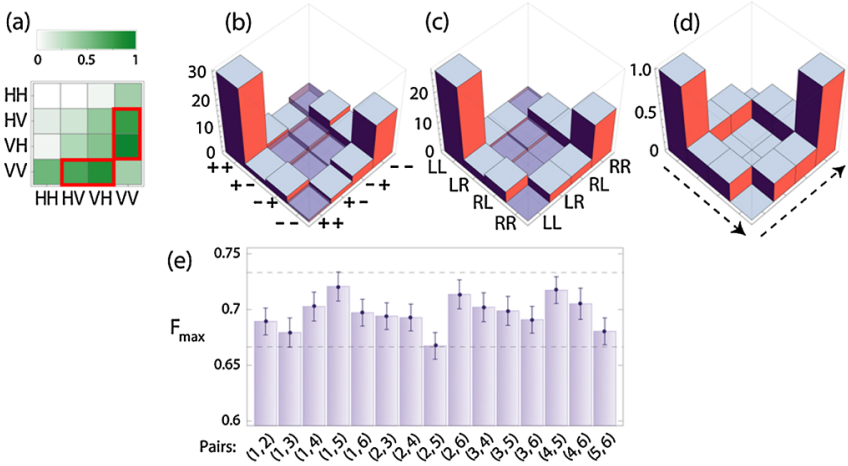

FIG. 4 (color online). Experimental data for the $W$ state $\varrho_{4}^{(1)}$. (a)-(c) Coincidence counts for $\varrho_{4}^{(1)}$ in the rescaled $|H / V\rangle,| \pm\rangle$, and $|L / R\rangle$ bases. (d) Ideal populations for (b) and (c). (e) Maximum achievable fidelity $F_{\max }$ using pair $(i, j)$ as a channel. The upper (lower) line shows the ideal (classical) value. 
use it for telecloning [11-13]. The fidelity limit for universal symmetric $1 \rightarrow(N-1)$ cloning is exactly $F_{\max }$ [13]; thus $\left|D_{N}^{(N / 2)}\right\rangle$ is an ideal resource for this task. Following [12], we have evaluated the protocol using $\varrho_{6}^{(3)}$ : Fig. 4(e) shows that the maximum cloning fidelity achievable is consistently above the classical threshold of $2 / 3$ [13]. A perfect $\left|\psi^{+}\right\rangle$channel for teleportation can be created, with success probability $\alpha_{N}$, if $N-2$ photons are measured out of $\left|D_{N}^{(N / 2)}\right\rangle$ in the $|H / V\rangle$ basis. This is in contrast to telecloning, where the photons are traced out, resulting in an imperfect channel with fidelity $\left\langle F_{\psi^{+}}\right\rangle_{\rho}=$ $\alpha_{N}$. As the core operation needed for telecloning commutes with the $\mid H$ or $V\rangle$ measurements, one can choose between telecloning and teleportation [11], with the success probability to teleport to any one party given by $p_{s}=$ $\alpha_{N} \geq \frac{1}{2}$. Thus $\left|D_{6}^{(3)}\right\rangle$ can be used for open-destination teleportation $[11,14]$. For $\varrho_{6}^{(3)}$ we find a mean value $\bar{p}_{s}=$ $0.55 \pm 0.02$ very close to the ideal $p_{s}=0.6$. As an example, we choose photons 5 and 6 , finding a mean fidelity $\left\langle\bar{F}_{\psi^{+}}\right\rangle_{\rho_{\exp }}=0.71 \pm 0.02$.

Finally, $\left|D_{N}^{(N / 2)}\right\rangle$ can also be used for multiparty quantum secret sharing [15], where entanglement ensures that all parties must cooperate in order to obtain a shared secret. The trick is to exploit the perfect correlations in the maximally conjugate bases of $\sigma_{x, y}$. Using $\left\langle\mathcal{C}_{i, j}^{\otimes N}(\theta)\right\rangle$, we get $\left\langle\sigma_{x, y}^{\otimes N}\right\rangle_{D_{N}^{(N / 2)}}=1$. Consider the $\sigma_{x}$ basis and $x_{j} \in\{0,1\}$ as the measurement outcome for the $j$ th photon. If photon 1 is measured, the value of $x_{1}$ can only be recovered via $x_{1}=$ $\oplus_{i=2}^{N} x_{i}$ ( $\oplus$ denotes mod-2 addition), implying cooperation of parties $2, \ldots, N$. As $\left|D_{N}^{(N / 2)}\right\rangle$ is symmetric, this applies to any choice for the initial party. The same holds for the $\sigma_{y}$ basis. When the parties announce their (randomly chosen) bases, a shared key can be distributed [15], with which a designated party encodes the secret. Any set of less than $N-1$ parties cannot recover the key, and although subsets of parties can exist with partial information about $x_{1}$ (for instance), any such bias is removable by postprocessing $[15,26]$. We thus evaluated the expected quantum bit error rate of the generated key (before postprocessing), given by the average error rate of the $\sigma_{x, y}$ bases. We find $25 \pm 2 \%$ and $29 \pm 1 \%$ for $N=4$ (415 shared bits) and $N=6(889$ shared bits), respectively, over an $82 \mathrm{~h}$ period.

Remarks. -We have demonstrated a linear-optics setup able to produce various states from the Dicke class and characterized their properties using new methods. We also evaluated the potential of our six-photon state for multiparty quantum networking. Our work significantly extends the range of attainable quantum states and paves the way toward the experimental study of other six-qubit entangled states [6] (and larger ones) and their use in quantuminformation processing.

We acknowledge discussions with J. Kofler, T. Jennewein, and N. Langford, help from B. Rauer, and support from EPSRC, QIPIRC, FWF, EC under the
Integrated Project Qubit Application, EMALI, and the U.S. Army Research Funded IARPA.

[1] R. Horodecki et al., Rev. Mod. Phys. 81, 865 (2009).

[2] D. M. Greenberger et al., Am. J. Phys. 58, 1131 (1990).

[3] W. Dür, G. Vidal, and J. I. Cirac, Phys. Rev. A 62, 062314 (2000).

[4] H. J. Briegel et al., Nature Phys. 5, 19 (2009).

[5] D. Bouwmeester et al., Phys. Rev. Lett. 82, 1345 (1999); M. Eibl et al., ibid. 90, 200403 (2003); Z. Zhao et al., Nature (London) 430, 54 (2004); H. Häffner et al., ibid. 438, 643 (2005); C.-Y. Lu et al., Nature Phys. 3, 91 (2007); W. Wieczorek et al., Phys. Rev. Lett. 101, 010503 (2008); W.-B. Gao et al., arXiv:0809.4277.

[6] F. Verstraete et al., Phys. Rev. A 65, 052112 (2002); L. Chen and Y.X. Chen, ibid. 74, 062310 (2006); L. Lamata et al., ibid. 75, 022318 (2007).

[7] R. H. Dicke, Phys. Rev. 93, 99 (1954).

[8] G. Tóth, J. Opt. Soc. Am. B 24, 275 (2007).

[9] A. Sørensen et al., Nature (London) 409, 63 (2001); J. K. Stockton et al., Phys. Rev. A 67, 022112 (2003); J. K. Korbicz, J. I. Cirac, and M. Lewenstein, Phys. Rev. Lett. 95, 120502 (2005); G. Tóth et al., ibid. 99, 250405 (2007); J. Korbicz et al., Phys. Rev. A 74, 052319 (2006).

[10] C. Thiel et al., Phys. Rev. Lett. 99, 193602 (2007); J. K. Stockton, R. van Handel, and H. Mabuchi, Phys. Rev. A 70, 022106 (2004); A. Retzker, E. Solano, and B. Reznik, ibid. 75, 022312 (2007); I. E. Linington and N. V. Vitanov, ibid. 77, 010302(R) (2008).

[11] N. Kiesel et al., Phys. Rev. Lett. 98, 063604 (2007).

[12] M. Murao et al., Phys. Rev. A 59, 156 (1999).

[13] V. Scarani et al., Rev. Mod. Phys. 77, 1225 (2005).

[14] C. H. Bennett et al., Phys. Rev. Lett. 70, 1895 (1993); A. Karlsson and M. Bourennane, Phys. Rev. A 58, 4394 (1998).

[15] M. Hillery, V. Bužek, and A. Berthiaume, Phys. Rev. A 59, 1829 (1999).

[16] A Dicke state of $N$ qubits and $k$ excitations is given by $\left|D_{N}^{(k)}\right\rangle=\left(C_{N}^{k}\right)^{-1 / 2} \sum_{P}|H\rangle^{\otimes k}|V\rangle^{\otimes N-k}$, with $C_{i}^{j}$ as the binomial coefficient.

[17] See EPAPS Document No. E-PRLTAO-103-082929. For more information on EPAPS, see http://www.aip.org/ pubservs/epaps.html.

[18] S. Campbell, M.S. Tame, and M. Paternostro, arXiv:0903.3939v1 [New J. Phys. (to be published)].

[19] Dephasing affecting $\left|D_{6}^{(3)}\right\rangle$ leads to only $(\sin \theta)^{6}$ [18].

[20] The LMSs are $\sigma_{x, y, z}^{\otimes 6}, \quad\left[\left(\sigma_{x} \pm \sigma_{y, z}\right) / \sqrt{2}\right]^{\otimes 6}, \quad\left[\left(\sigma_{y} \pm\right.\right.$ $\left.\left.\sigma_{z}\right) / \sqrt{2}\right]^{\otimes 6},\left[\left(\sigma_{x, y} \pm 2 \sigma_{z}\right) / \sqrt{5}\right]^{\otimes 6}, \quad\left[\left(\sigma_{z} \pm 2 \sigma_{x, y}\right) / \sqrt{5}\right]^{\otimes 6}$, and $\left[\left(\sigma_{x} \pm \sigma_{y} \pm \sigma_{z}\right) / \sqrt{3}\right]^{\otimes 6}$.

[21] In the $\sigma_{x, y}$ bases, for instance, $\left|D_{6}^{(3)}\right\rangle$ has an overlap of $\sqrt{5 / 8}$ with a six-photon $\mathrm{GHZ}$ state.

[22] This was independently investigated in W. Wieczorek et al., Phys. Rev. A 79, 022311 (2009).

[23] The LMSs are $\sigma_{x, y, z}^{\otimes 4}, \quad\left[\left(\sigma_{x} \pm \sigma_{y, z}\right) / \sqrt{2}\right]^{\otimes 4}, \quad\left[\left(\sigma_{y} \pm\right.\right.$ $\left.\left.\sigma_{z}\right) / \sqrt{2}\right]^{\otimes 4}$

[24] O. Gühne et al., Phys. Rev. A 76, 030305(R) (2007).

[25] M. Horodecki, P. Horodecki, and R. Horodecki, Phys. Rev. A 60, 1888 (1999).

[26] S. Gaertner et al., Phys. Rev. Lett. 98, 020503 (2007). 